\title{
Dynamical evolution of rotating globular clusters with embedded black holes
}

\author{
José Fiestas and Rainer Spurzem \\ Zentrum für Astronomie, Astronomisches Rechen-Institut, Heidelberg, Germany \\ email: fiestas, spurzem@ari.uni-heidelberg.de
}

\begin{abstract}
Evolution of rotating globular clusters with embedded black holes is presented. The interplay between velocity diffusion due to relaxation and black hole star accretion is followed together with cluster rotation, using 2-dimensional, in energy and z-component of angular momentum, Fokker Planck numerical methods. Gravogyro and gravothermal instabilities drive the system to a faster evolution leading to shorter collapse times and a faster cluster dissolution in the tidal field of a parent galaxy.

Angular momentum transport and star accretion support the development of central rotation in relaxation time scales. Two-dimensional distribution (in the meridional plane) of kinematical and structural parameters (density, dispersions, rotation) are reproduced, with the aim to enable the use of set of models for comparison with observational data.
\end{abstract}

Keywords. Gravitation - stellar dynamics - globular clusters: general - black hole physics

The stellar system is assumed to be axisymmetric and in dynamical equilibrium. We use initially rotating King models of the form:

$$
f_{\mathrm{rk}}\left(E, J_{z}\right)=\mathrm{const}[\exp (-\beta E)-1] \exp \left(-\beta \Omega_{0} J_{z}\right)
$$

$\omega_{0}=\sqrt{9 / 4 \pi G n_{\mathrm{c}}} \cdot \Omega_{0}$ is the dimensionless angular velocity, and a modified evolving potential $\phi(t)=\phi_{\mathrm{cl}}(t)-G M_{b h}(t) / r$, where $G=M_{\mathrm{i}}=r_{\mathrm{c}_{\mathrm{i}}}=1$. Using an initial $M_{\mathrm{bh}}=5 \times 10^{-5} M_{\mathrm{cl}}$, we followed the evolution up to core collapse, where $M_{\mathrm{bh}} \sim 0.1 M_{\mathrm{cl}}$ was found. We confirmed the acceleration of core collapse in single-mass rotating systems with $\mathrm{BH}$ with respect to the non-rotating case. At collapse time, the BH-potential dominates the stellar distribution inside the influence radius $\left(r_{a}\right)$. The density and velocity dispersion distributions evolve to a power-law of $n \propto r^{\lambda}, \quad \lambda=-1.75$ and $\sigma \propto r^{\gamma}, \gamma=-0.5$ (Bahcall \& Wolf (1976), Marchant \& Shapiro (1980)). Post-collapse is driven through energy input from the central object. The rotational velocity and velocity dispersion distributions in the meridional plane reproduce the observed morphological structure of rotating clusters (see for example non-parametric fitting of kinematical data of $\omega$ Cen in Merritt et al., (1997), and compared to our data in Fiestas, Spurzem \& Kim (2006)). Rotational velocity grows inside the $\mathrm{BH}$ influence radius strong influenced by the interplay between diffusion of angular momentum (gravogyro instability) and the redistribution of high energy, low $J_{z}$ orbits close to the BH (loss-cone effect).

The models presented, are able to reproduce 2D distributions of kinematical and structural parameters, at any time of evolution and deep in the stellar cusp surrounding the central BH. Observational studies of globular clusters can be compared to evolutionary models, to elucidate theoretical predictions and understand the common evolution of star clusters and galaxies. With this aim, a detailed set of model data, covering a wide range of rotation rates and initial concentrations of rotating $\mathrm{GCs}$, with and without $\mathrm{BH}$, has been 

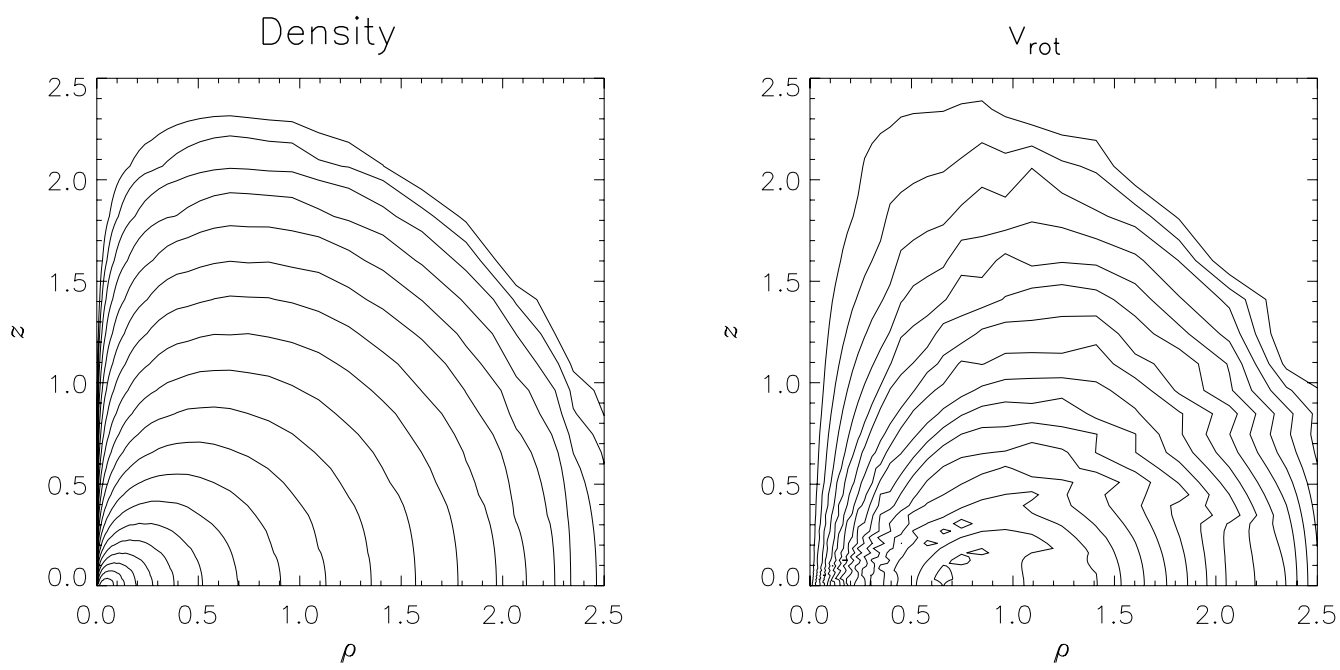

Figure 1. 2D density (left) and rotational velocity distribution (right) in the meridional plane for an initial King model $\left(W_{0}=6.0, \omega_{0}=0.9\right)$, at time $t / t_{\mathrm{rhi}}=3.45$. The $\mathrm{BH}\left(M_{\mathrm{bh}} \sim 0.01 M_{\mathrm{cl}}(0)\right)$ is located at the center of coordinates. Note the influence of rotation in the density distribution.

created, to enable observers to use them for comparisons with their data. The cluster database can be found on the web, at http://www.ari.uni-heidelberg.de/clusterdata/.

The influence of rotation in the evolution and redistribution of orbits in the cluster is illustrated in Fig. 1, for a high rotating model $\left(\omega_{0}=0.9\right)$ at a late stage of evolution $\left(t / t_{\mathrm{rhi}}=3.45\right)$. Observe the increase of central rotation in torus-like contours of density and $v_{\text {rot }}$.

Although our models agree with published theoretical studies of dense stellar systems in the spherical case and with the observed BH-mass in globular clusters, our models are still idealized and simplified. Physical scalings, such as time scales, may change if more effects such of a mass spectrum and binaries are included (items partly in work).

\section{Acknowledgements}

We thank the Local Organizing Committee and ZAH Heidelberg for the financial support.

\section{References}

Bahcall, J. N. \& Wolf, R. A. 1976, ApJ, 209, 214

Fiestas, J., Spurzem, R. \& Kim, E. 2006, MNRAS, 373, 677

Marchant, A. B. \& Shapiro, S. L. 1980, ApJ, 239, 685

Merrit, D. et al. 1997, ApJ, 114, 1074 